Research

Open Access

\title{
Changes in regional distribution of lung sounds as a function of positive end-expiratory pressure
}

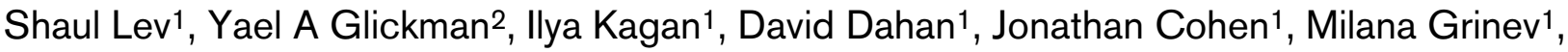 \\ Maury Shapiro ${ }^{1}$ and Pierre Singer ${ }^{1}$
}

\author{
1Department of General Intensive Care, Rabin Medical Center, Beilinson Campus, 39 Jabotinski Street., Petach Tikva, 49100, Israel \\ 2Deep Breeze, Ltd., 2 Hailan St., P.O. Box 140, North Industrial Park, Or-Akiva, 30600, Israel \\ Corresponding author: Shaul Lev, lev.nirit@gmail.com \\ Received: 7 Nov 2008 Revisions requested: 16 Jan 2009 Revisions received: 27 Apr 2009 Accepted: 10 May 2009 Published: 10 May 2009 \\ Critical Care 2009, 13:R66 (doi:10.1186/cc7871) \\ This article is online at: http://ccforum.com/content/13/3/R66 \\ (c) 2009 Lev et al.; licensee BioMed Central Ltd. \\ This is an open access article distributed under the terms of the Creative Commons Attribution License (http://creativecommons.org/licenses/by/2.0), \\ which permits unrestricted use, distribution, and reproduction in any medium, provided the original work is properly cited.
}

\begin{abstract}
Introduction Automated mapping of lung sound distribution is a novel area of interest currently investigated in mechanically ventilated, critically ill patients. The objective of the present study was to assess changes in thoracic sound distribution resulting from changes in positive end-expiratory pressure (PEEP). Repeatability of automated lung sound measurements was also evaluated.

Methods Regional lung sound distribution was assessed in 35 mechanically ventilated patients in the intensive care unit (ICU). A total of 201 vibration response imaging (VRI) measurements were collected at different levels of PEEP between 0 and 15 $\mathrm{cmH}_{2} \mathrm{O}$. Findings were correlated with tidal volume, oxygen saturation, airway resistance, and dynamic compliance. Eightytwo duplicated readings were performed to evaluate the repeatability of the measurement.
\end{abstract}

Results A significant shift in sound distribution from the apical to the diaphragmatic lung areas was recorded when increasing PEEP (paired t-tests, $P<0.05$ ). In patients with unilateral lung pathology, this shift was significant in the diseased lung, but not as pronounced in the other lung. No significant difference in lung sound distribution was encountered based on level of ventilator support needed. Decreased lung sound distribution in the base was correlated with lower dynamic compliance. No significant difference was encountered between repeated measurements.

Conclusions Lung sounds shift towards the diaphragmatic lung areas when PEEP increases. Lung sound measurements are highly repeatable in mechanically ventilated patients with various lung pathologies. Further studies are needed in order to fully appreciate the contribution of PEEP increase to diaphragmatic sound redistribution.

\section{Introduction}

The use of acoustic monitoring technology offers the potential for a radiation-free, noninvasive bedside assessment of lung abnormality in patients during their stay in the intensive care unit (ICU). Correlation between breath sound recordings and regional distribution of pulmonary ventilation has been previously established, particularly in studies conducted by PloySong-Sang and colleagues and other groups who compared acoustic findings with data obtained with radioactive gases [13]. The effect of airflow and volume on the amplitude and spectral content of breath sounds has been extensively stud- ied in healthy [4-9] and diseased lungs [10-12]. Furthermore, several studies assessed the effect of changes of mechanical ventilation on lung sound distribution in animal models [1317]. Räsenen and colleagues reported that the acoustic changes associated with oleic acid-induced lung injury allow monitoring of its severity and distribution [13] and that acute lung injury (ALI) causes regional acoustic transmission abnormalities that are reversed during alveolar recruitment with positive end-expiratory pressure (PEEP) [14]. Recently, Vena and colleagues reported a reduction of amplitude and a change in spectral characteristics of normal lung sounds when increas-

ADR: apico-diaphragmatic ratio; ALI: acute lung injury; ARDS: acute respiratory distress syndrome; Cdyn: dynamic compliance; CV: coefficients of variation; $\mathrm{FiO}_{2}$ : fraction of inspired oxygen; ICU: intensive care unit; LL: lower left; LR: lower right; $\mathrm{ML}$ : middle left; $\mathrm{MR}$ : middle right; PaO 2 : partial arterial pressure of oxygen; PEEP: positive end-expiratory pressure; PSV: pressure support ventilation mode; R²: coefficients of determination; Raw: airway resistance; RR: respiratory rate; $\mathrm{SpO}_{2}$ : oxygen saturation; SIMV: synchronized intermittent mandatory ventilation; TL: total left lung; TR: total right lung; UL: upper left; UR: upper right; VRI: vibration response imaging; VT: tidal volume. 
ing PEEP in mechanically ventilated pigs [15]. Finally, recording of crackle-sound during mechanical ventilation was employed to monitor lung recruitment-derecruitment in a porcine model $[16,17]$.

The experience on acoustic monitoring in mechanically ventilated patients is limited $[18,19]$ and only preliminary investigations were conducted to assess changes in regional distribution of lung sound as a function of changes in mechanical ventilator setting $[20,21]$. Waitman and colleagues classified breath sounds recorded in an intensive care setting using different neural network configurations [22], and a computerized respiratory sound monitor was used to detect wheezes in pediatric ICU [23]. Detection of endobronchial [24-26] and esophageal [27] intubation using lung sound monitoring during anesthesia was also described. Dellinger and colleagues recently reported the use of an acoustic-based imaging device to map the geographical distribution of breath sound as a function of mechanical ventilation mode [28]. Changes in lung sound distribution map during recruitment maneuver and PEEP increase were also reported in four abstracts [29-32]. These findings suggest that breath sound information can be used to evaluate lung condition during mechanical ventilation; however, information regarding lung sound monitoring to adjusted PEEP levels is lacking. PEEP setting is widely used by physicians and respiratory therapists in order to improve gas exchange, mainly in patients with severe hypoxic respiratory failure such as acute respiratory distress syndrome (ARDS) and ALI [33] while preventing end-expiratory alveolar collapse [34] and inspiratory overinflation [35]. In practice PEEP setting is adjusted to patient condition up to several times a day, although no standardized method to adjust PEEP has been accepted to date. The first step in the evaluation of a new approach is to assess if a change in PEEP induces any change in the measurement.

The aim of the present study was to evaluate the effect of changes in PEEP on the regional distribution of lung sounds as recorded by vibration response imaging (VRI), an acoustic monitoring technology that creates a dynamic two-dimensional functional image of lung sound distribution during mechanical ventilation. Repeatability of lung sound measurements was also evaluated.

\section{Materials and methods Patients}

The study was performed in the general ICU of the Rabin Medical Center in Petach-Tikva, Israel. The study protocol was approved by the Institutional Review Board and informed consent was obtained from all patients or their next-of-kin. Intraindividual differences in lung sound measurements were investigated at different levels of PEEP in a prospective trial.

\section{Inclusion and exclusion criteria}

Patients enrolled in the study were adults (18 to 85 years old) with a body mass index greater than 21. Exclusion criteria included a body habitus or skin condition that would interfere with sensor placement; the presence of a cardiac pacemaker, implantable defibrillator or artificial heart valve; or pregnancy.

\section{Study design}

The levels of PEEP and the fraction of inspired oxygen $\left(\mathrm{FiO}_{2}\right)$ were adjusted according to clinical requirements. Among the 35 patients enrolled in this study, one was recorded at PEEP 5 and $10 \mathrm{cmH}_{2} \mathrm{O}$ and 34 at PEEP 0,5 and $10 \mathrm{cmH}_{2} \mathrm{O}$. Fifteen of these 34 patients were also recorded at PEEP $15 \mathrm{cmH}_{2} \mathrm{O}$. In 28 patients, PEEP was assigned from low to high level. In order to assess any effect due to the lack of randomization, PEEP levels were applied in a random order in a subgroup of patients $(n=7)$. At the later stage of the protocol, repeatability was tested on 26 patients for whom two repeated consecutive measurements were performed at the same level of PEEP under the same conditions, over a period of time not exceeding five minutes. No recording was excluded from the repeatability study. Measurements at different PEEP levels were performed at an interval of at least five minutes. No intervention, except for changes in PEEP, was allowed by the protocol. Mode of mechanical ventilation, tidal volume (VT), respiratory rate (RR), partial arterial pressure of oxygen $\left(\mathrm{PaO}_{2}\right), \mathrm{FiO}_{2}$, oxygen saturation $\left(\mathrm{SpO}_{2}\right)$, airway resistance (Raw) and online dynamic compliance (Cdyn) as provided by the ventilator were documented. Three consecutive measurements of $\mathrm{Cdyn}$ were averaged in order to reduce variability. To keep consistency and ensure that timing between spontaneous and controlled cycles do not affect the results, the spontaneous breath was used whenever available (31 out of 35 patients, 89\%), including in synchronized intermittent mandatory ventilation mode (SIMV).

\section{Recording procedure}

A schema of the apparatus is provided in Figure 1. The recordings were performed using a VRI $x^{\mathrm{TM}}$ device (Deep Breeze Ltd., Or-Akiva, Israel) with two arrays of six rows by three columns sensors or microphones similar to those used in digital stethoscopes. The recordings were made in supine position with a bed angle between 30 to $45^{\circ}$. The arrays were positioned posterior to the patient's back using a disposable positioning unit to reduce risk of cross-contamination. Morphological landmarks such as spine and scapula were used in order to ensure accurate and repeatable placement of the sensor arrays. Excessive secretions were removed by endotracheal and oral suctioning before each series of recordings. Airway pressure and flow waveforms were sampled from the ventilator using a proximal flow sensor inserted in the patient's circuit. As displayed in Figure 2, these waveforms were synchronized with the sound energy graph representing the average sound energy in both lungs. Each recording lasted for 20 seconds of acquisition time, followed by 40 seconds of 


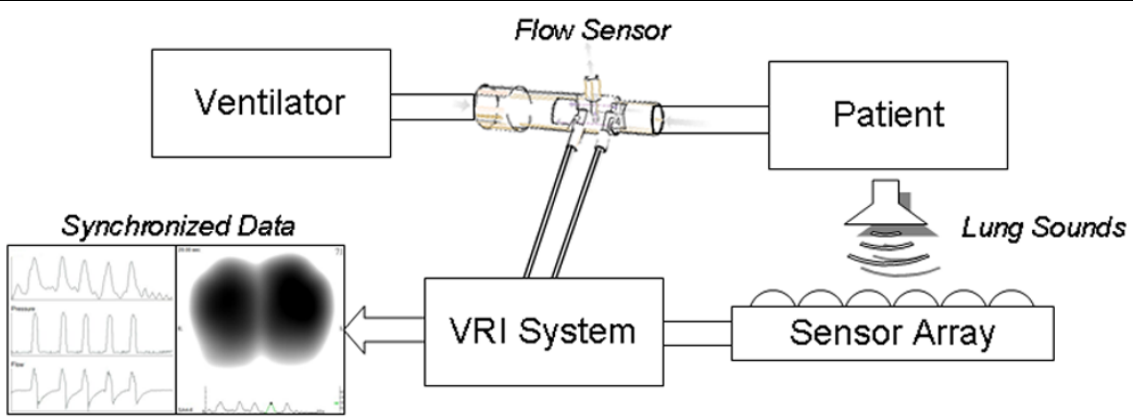

Schematic diagram describing the elements of the system. The patient lies on the acoustic sensor array and the flow sensor is inserted in the breathing circuit. The vibration response imaging (VRI) system collects acoustic information simultaneously from the sensor array and pressure and flow waveforms from the ventilator.

processing time, and was stored digitally on the device for later review and analysis.

\section{Measurement output}

The output of the measurement consisted of a normalized dynamic image synchronized with airway pressure and flow waveforms, revealing the geographical distribution of sound energy during the respiratory cycle. Because of image normalization, the measurement output aimed at describing the relative airflow distribution in the lung rather than the absolute volume. As described in detail by Dellinger and colleagues [36], this dynamic image was created from a series of grayscale still images or frames with each frame representing 0.17 seconds of sound energy. The digitized acoustic signals were band-pass filtered between 150 and $250 \mathrm{~Hz}$ to remove heart and muscle sounds; median filtering was applied to suppress impulse noise, and truncation of samples above an automatically determined signal-to-noise threshold was performed. Sound energy was obtained following down-sampling.

Recording quality was assessed according to pre-determined criteria [28]. The graph representing the average sound energy as a function of time throughout the respiratory cycle in both lungs was displayed underneath the dynamic image. Each 20 second measurement included up to 10 respiratory cycles. A normalized representative frame (or map) at peakinspiratory flow was automatically selected and displayed on the screen (Figure 2). This map was also quantified by the software and presented as the percentage of weighted pixels in six lung regions: upper right (UR); middle right (MR); lower right (LR); upper left (UL); middle left (ML) and lower left (LL), up to a total of $100 \%$. According to the recording procedure,

Figure 2

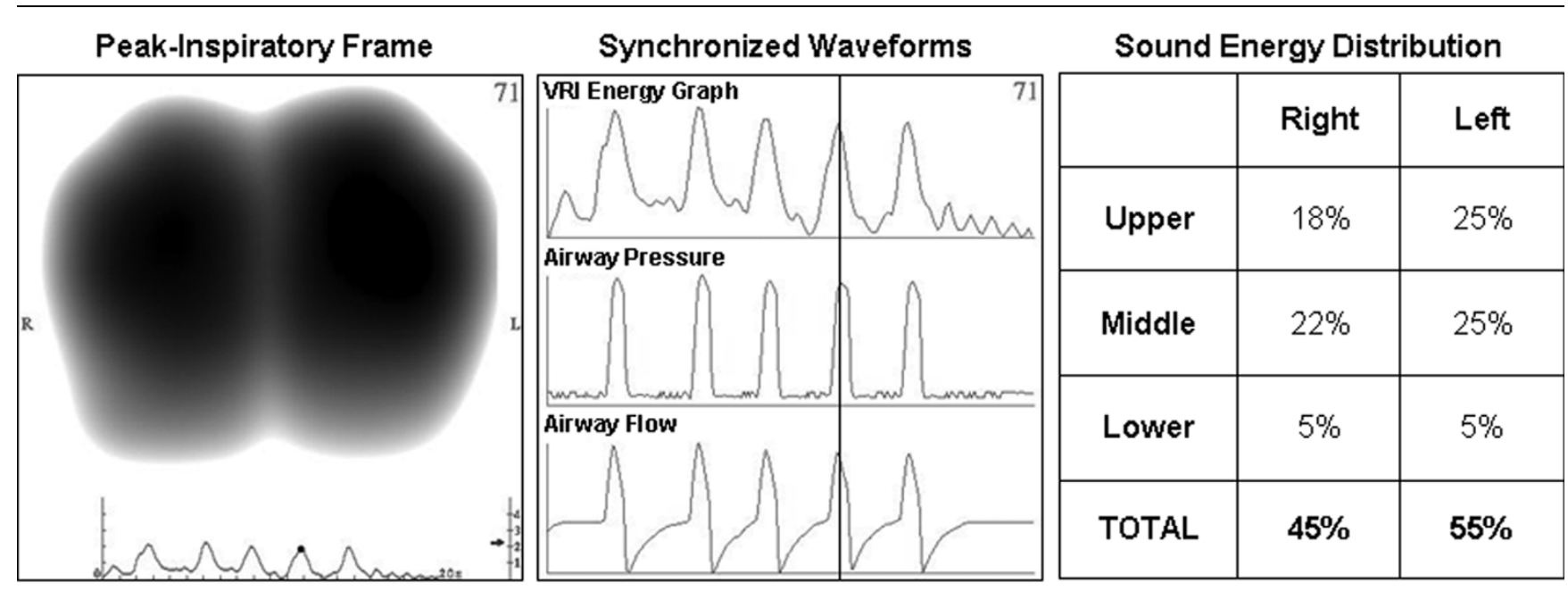

An example of acoustic data as displayed for a recording obtained from a 77-year-old male with myasthenia gravis. A representative peak-inspiratory image (left panel); synchronized sound energy graph and ventilator airway pressure and flow waveforms (middle panel); sound energy distribution in the six lung regions as automatically provided by the software in percentage of weighted pixel count (right panel). VRI = vibration response imaging. 
'lower' corresponded to the diaphragmatic lung region and 'upper' to the apical lung region. The percentage of sound energy in the total right lung (TR $=U R+M R+L R)$ and total left lung ( $T L=U L+M L+L L)$ were also documented. The apico-diaphragmatic ratio (ADR), defined as the ratio between the lung sound distribution in the apical lung areas (UR + UL) and the diaphragmatic lung areas (LR + LL) (ADR $=(\mathrm{UL}+$ $\mathrm{UR}) /(\mathrm{LL}+\mathrm{LR})$ ), was used to assess changes in lung sound distribution along the cephalocaudal axis. A larger ratio suggested increased sound in the apical areas and a smaller ratio increased sound in the diaphragmatic areas. Distribution was considered more heterogeneous if the difference was larger than two; this threshold was derived from experience with healthy patients in supine positions.

\section{Statistical analysis}

Data are presented as the mean \pm standard deviation. When two recordings were performed under identical conditions, the second recording was used for all analysis not related to the repeatability aspect of the study. For samples including more than 30 measurements, paired student's t-test (Microsoft ${ }^{\circledR}$ Office Excel 2003, Microsoft Corporation, Redmond, WA, USA) was used. For the analysis of samples including less than 30 measurements, Wilcoxon matched-pairs signed-ranks test, Friedman two-way analysis on ranks test and Wilcoxon two sample test were used (IFA Services Statistics, Amsterdam, Holland). Friedman test was used to compare three or more paired groups. Coefficients of determination $\left(R^{2}\right)$ and coefficients of variation (CV) were used to test repeatability. A $P<0.05$ was considered significant.

\section{Results}

A total of 35 mechanically ventilated patients (26 males, 9 females, age $62 \pm 20$ years) were enrolled in the study between April 2007 and January 2008. Patients were ventilated using one of two types of ventilators (Puritan Bennett, Tyco Healthcare, Mansfield, MA, USA; Evita XL or Evita 4, Draeger, Lübeck, Germany). The majority of the patients ( $n=$ $26 ; 74 \%$ ) were mechanically ventilated on pressure support ventilation mode (PSV) with a level between 8 and $24 \mathrm{cmH}_{2} \mathrm{O}$ (mean $14 \pm 4 \mathrm{cmH}_{2} \mathrm{O}$ ). Six patients (17\%) were ventilated using SIMV. The rest of the patients $(n=3 ; 8 \%)$ were ventilated with other modes of mechanical ventilation. Patients were not deeply sedated and none were paralyzed.

A total of 201 valid recordings were performed on the 35 patients. No adverse event related to the measurement was registered. Ten recordings (less than $5 \%$ of the overall data) were excluded from the analysis based on pre-determined criteria as mentioned above [28]. Poor recording quality was confirmed by an average sound energy level below a predefined threshold $<1$ in the energy bar of the imaging display). Reasons for mechanical ventilation of these 35 patients are described in Table 1. Chest radiography results revealed that 19 of these patients had bilateral disease, 13 had unilat-
Table 1

\begin{tabular}{lcc}
\hline Reason for mechanical ventilation in 35 patients & & \\
\hline \multicolumn{1}{c}{ Reason for intubation } & N & $\%$ \\
\hline Pneumonia & 8 & 23 \\
Acute respiratory failure & 7 & 20 \\
Severe chest trauma & 3 & 9 \\
Interstitial lung disease & 2 & 6 \\
Cerebrovascular accident & 2 & 6 \\
Congestive heart failure & 2 & 6 \\
Acute respiratory distress syndrome & 2 & 6 \\
Pancreatitis & 2 & 6 \\
Head trauma & 2 & 6 \\
Others* & 5 & 14 \\
\hline
\end{tabular}

*Sepsis, chronic obstructive pulmonary disease, myasthenia gravis, failure to wean, mesenterial ischemia.

eral lung pathology inducing decreased lung sounds (i.e. onelung atelectasis, pneumothorax, or pleural fluid) and three had normal lungs. Average VT was $551 \pm 126 \mathrm{~mL}, \mathrm{SpO}_{2} 97 \pm 3 \%$, RR $21 \pm 7$ breaths/minute, Cdyn $60 \pm 42 \mathrm{~mL} / \mathrm{mbar}$, and Raw $16 \pm 5 \mathrm{mbar} \mathrm{L} / \mathrm{second}$. These parameters did not significantly change with PEEP (Friedman test, paired groups).

Paired analysis conducted on the 34 patients for which recordings at PEEP 0,5 , and $10 \mathrm{cmH}_{2} \mathrm{O}$ were available revealed that the proportion of sound energy in the diaphragmatic lung regions (LR and $L L$ ) was significantly increased with PEEP ( $P<0.05$, paired t-test), while the proportion of sound energy in the apical lung regions (UR and UL) was decreased ( $P<0.05$ in UL, paired t-test). The proportion of energy in the middle areas of the lungs (MR and $M L)$ did not significantly change with PEEP (Figure 3 ). No additional shift was detected at PEEP $15 \mathrm{cmH}_{2} \mathrm{O}(\mathrm{n}=15$, Wilcoxon matched paired test). In patients with unilateral lung pathology $(n=13)$, the increase in sound energy in the diaphragmatic lung regions was significant in the diseased lung ( $7 \pm 6 \%$ at $P E E P$ $0 \mathrm{cmH}_{2} \mathrm{O}$ versus $10 \pm 7 \%$ at PEEP $10 \mathrm{cmH}_{2} \mathrm{O}, P=0.01$, Wilcoxon matched-pairs) but not significant in the other lung (14 $\pm 8 \%$ at $\mathrm{PEEP} O \mathrm{cmH}_{2} \mathrm{O}$ versus $15 \pm 9 \%$ at PEEP $10 \mathrm{cmH}_{2} \mathrm{O}$, Wilcoxon matched-pairs). In patients with bilateral lung pathology $(\mathrm{n}=21)$, the increase was significant in both lungs $(P=$ 0.04).

The majority of the patients were ventilated on PSV or SIMV, spontaneous diaphragmatic activity was present in most of the patients. In order to assess the extent of this confounding factor, analysis was conducted according to the level of ventilator support provided to the patients. Patients were divided into two subsets according to the level of ventilator support needed (PSV $<15 \mathrm{cmH}_{2} \mathrm{O}$ and PSV $>15 \mathrm{cmH}_{2} \mathrm{O}$ ). Sound energy distribution was compared between the two groups at 


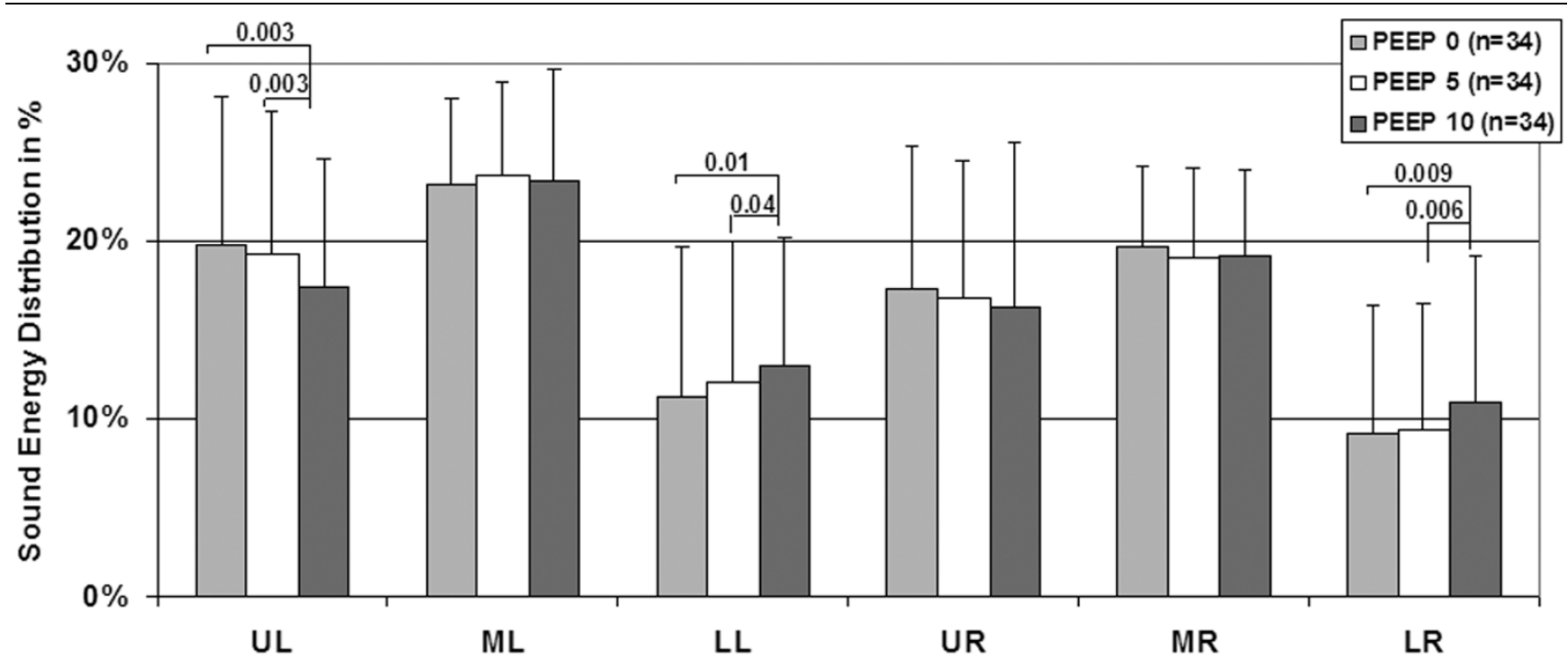

Mean \pm standard deviation of sound energy distribution in 34 mechanically-ventilated patients recorded at three levels of PEEP ( 0,5 and 10 $\mathrm{cmH}_{2} \mathrm{O}$ ). Significant $P$ values are indicated (paired t-tests). $\mathrm{LL}=$ lower left; $\mathrm{LR}=$ lower right; $\mathrm{ML}=$ middle left; $\mathrm{MR}=$ middle right; $\mathrm{PEEP}=$ positive end-expiratory pressure; UL = upper left; UR = upper right.

each level of PEEP. No significant difference was detected (Wilcoxon two sample tests).

As shown in Figure 4, per patient analysis revealed that when increasing PEEP from 0 to $10 \mathrm{cmH}_{2} \mathrm{O}$, sound energy distribution increased in the diaphragmatic lung areas in $76 \%$ of the patients (26 of 34). In these cases, a larger peak-inspiratory flow image was obtained at higher PEEP (examples in Figure $5 \mathrm{a}$ and $5 \mathrm{~b}$ ). In several patients, an asymmetrical change of lung sound energy distribution was recorded at PEEP 15 $\mathrm{CmH}_{2} \mathrm{O}$ (Figure 5c, d, and 5e). Comparisons between VT, $\mathrm{SpO}_{2}$, Cdyn and Raw at two different levels of ADR are summarized in Table 2. When adjusted for RR, no difference in VT, Raw and $\mathrm{SpO}_{2}$ was encountered between the two levels of ADR. At RRs lower than 20 breaths/minute, Cdyn tended to be higher for recordings with increased energy in the lower lung regions $(A D R<2)$. This difference approached significance $(P=0.058)$.

The repeatability of the measurement was assessed in 82 sets of double recordings obtained from 26 patients (20 double recordings at PEEP $0 \mathrm{cmH}_{2} \mathrm{O} ; 25$ at PEEP $5 \mathrm{cmH}_{2} \mathrm{O} ; 26$ at PEEP $10 \mathrm{cmH}_{2} \mathrm{O}$, and 11 at PEEP $15 \mathrm{cmH}_{2} \mathrm{O}$ to a total of 164 recordings). Repeatability was performed by comparing the distribution of sound energy in each of the six lung regions of two repeated measurements, as well as in total left and right lungs. No significant difference was encountered between repeated measurements (paired t-test). Mean $\mathrm{R}^{2}$ obtained for the different lung regions was $0.93 \pm 0.02$ (range 0.91 to 0.95 ) with a CV equal to $1.7 \%$.

\section{Discussion}

In this study, we used an acoustic-based monitoring system in order to assess possible shift in thoracic sound distribution during PEEP changes and to evaluate the repeatability of lung sound measurements in mechanically ventilated patients. Our results revealed a significant increase in sound distribution from the apical to the diaphragmatic lung areas when increasing PEEP from 0 to $10 \mathrm{cmH}_{2} \mathrm{O}$. This shift was especially pronounced in patients with severe lung pathology but was not affected by the level of pressure support needed. These statistical results were further supported by the analysis of the effect of PEEP on lung sound distribution in individual patients. As revealed in Figure 4, lung sound increased in the diaphragmatic lung areas in $76 \%$ of the patients.

The explanation for this acoustic phenomenon might be related to an increase in ventilation distribution in the diaphragmatic part of the lungs at higher levels of PEEP or to the effect of other PEEP-related physiologic factors, such as translocation of fluid from alveolar to interstitial spaces. A similar shift of lung sound distribution towards the base was recently described by Dellinger and colleagues [28], while changing mode of mechanical ventilation from volume control to pressure control and pressure support. The authors speculated that this shift was produced by a diaphragm-generated negative intrapleural pressure in pressure-targeted modes. The authors also proposed that the initial higher flow in pressuretargeted modes may serve to prime the proximal airway, allowing more time for slower, more laminar flow to produce a more homogenous distribution of air to lower lung regions. Correla- 


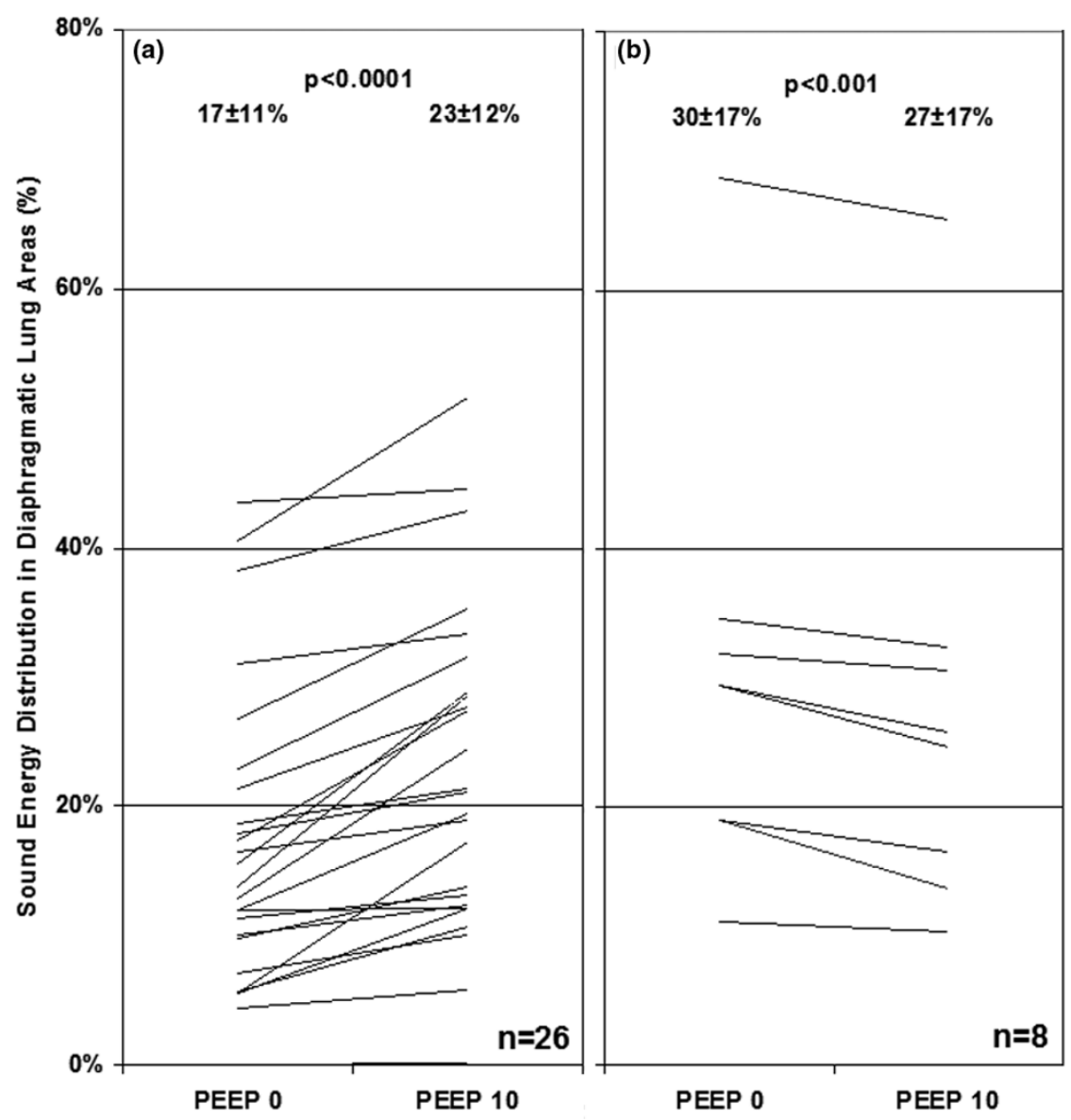

Individual sound energy distribution in diaphragmatic lung areas in 34 mechanically-ventilated patients recorded at $\mathrm{PEEP}$ levels 0 and $10 \mathrm{cmH} \mathrm{H}_{2} \mathrm{O}$. Sound energy distribution increased from $17 \pm 11 \%$ to $23 \pm 12 \%(P<0.0001)$ in (a) 26 'responder' patients and decreased from $30 \pm 17 \%$ to 27 $\pm 17 \%(P<0.001)$ in $(b)$ eight 'non-responder' patients. $P E E P=$ positive end-expiratory pressure.

tion between lung condition and heterogeneity of lung sound distribution has been described in several additional studies. Bentur and colleagues [37] identified greater heterogeneity of lung sound distribution in pediatric patients with confirmed cases of foreign body aspiration when compared with healthy subjects. Lung sound heterogeneity was also described by Jean and colleagues [38] when comparing measurements performed on patients with normal lungs versus one diseased lung on chest radiograph. In agreement with the image normalization method used to generate the lung acoustic map, the authors explained that a larger image was considered to indicate a more homogeneous sound distribution and a smaller image a more focal distribution. In our study, the shift of lung sound distribution towards the base at PEEP $10 \mathrm{cmH}_{2} \mathrm{O}$ was accompanied by an increase in the size of the peak-inspiratory flow image, in line with increased homogeneity of lung sound distribution. At PEEP level of $15 \mathrm{cmH}_{2} \mathrm{O}$, however, the lack of increased shift towards the base was adjunctive with a decrease in the size of the image as exemplified in Figures $5 \mathrm{c}$ to $5 \mathrm{e}$. In light of the effect of PEEP elevation in lung sound dis- tribution, comparison between measurements should be preferably performed when similar PEEP levels are applied.

Repeatability of the lung acoustic measurements was comparable with that reported in healthy subjects $[8,39,40]$. This result in patients mechanically ventilated in pressure support mode may be a priori unexpected, especially when considering the variability of VT anticipated in this mode. However, this finding confirms that normalization of the acoustic distribution map reduces the mere effect of changes in ventilator settings when these changes do not affect the relative airflow distribution. Figure $6 \mathrm{a}$, representing normalized images recorded from the same patient ventilated with two different VT, and Figure $6 \mathrm{~b}$, representing normalized images recorded from the same patient ventilated with two different airflow rates, further illustrate this finding.

The scope of this study was limited because of a restrictive protocol. Enrollment of deeply sedated patients mechanically ventilated in volume-controlled mode of mechanical ventilation 
(a)
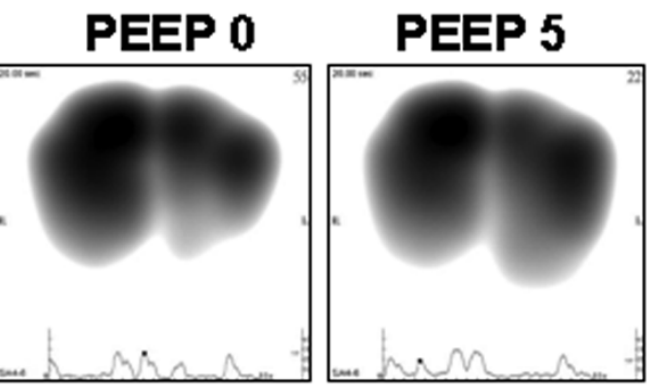

\section{P}

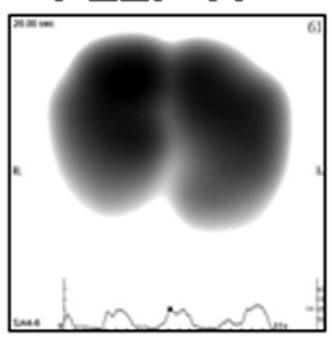

(b)
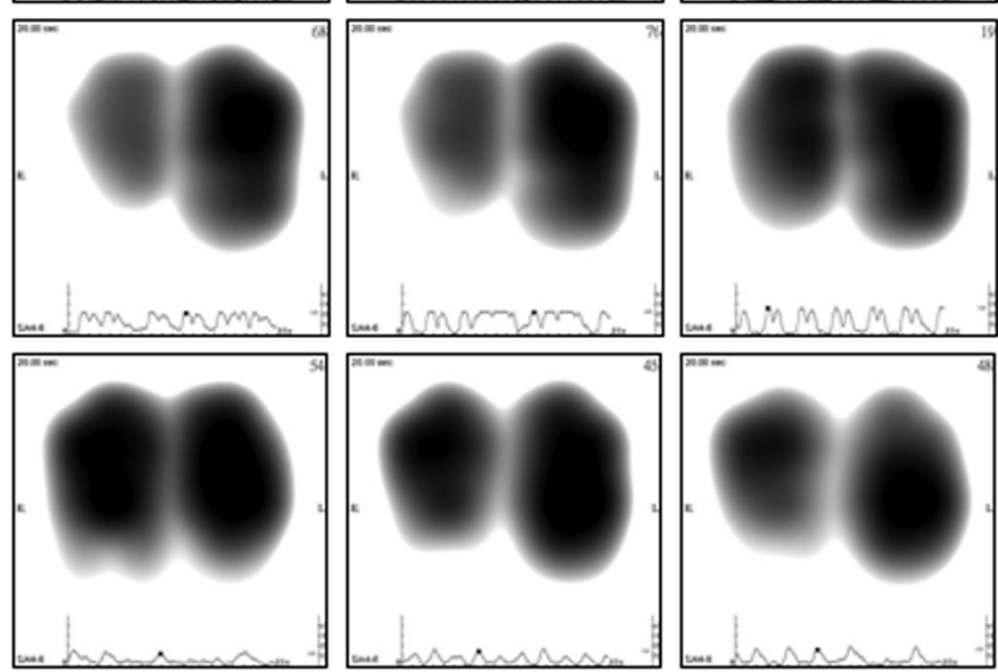

(c)
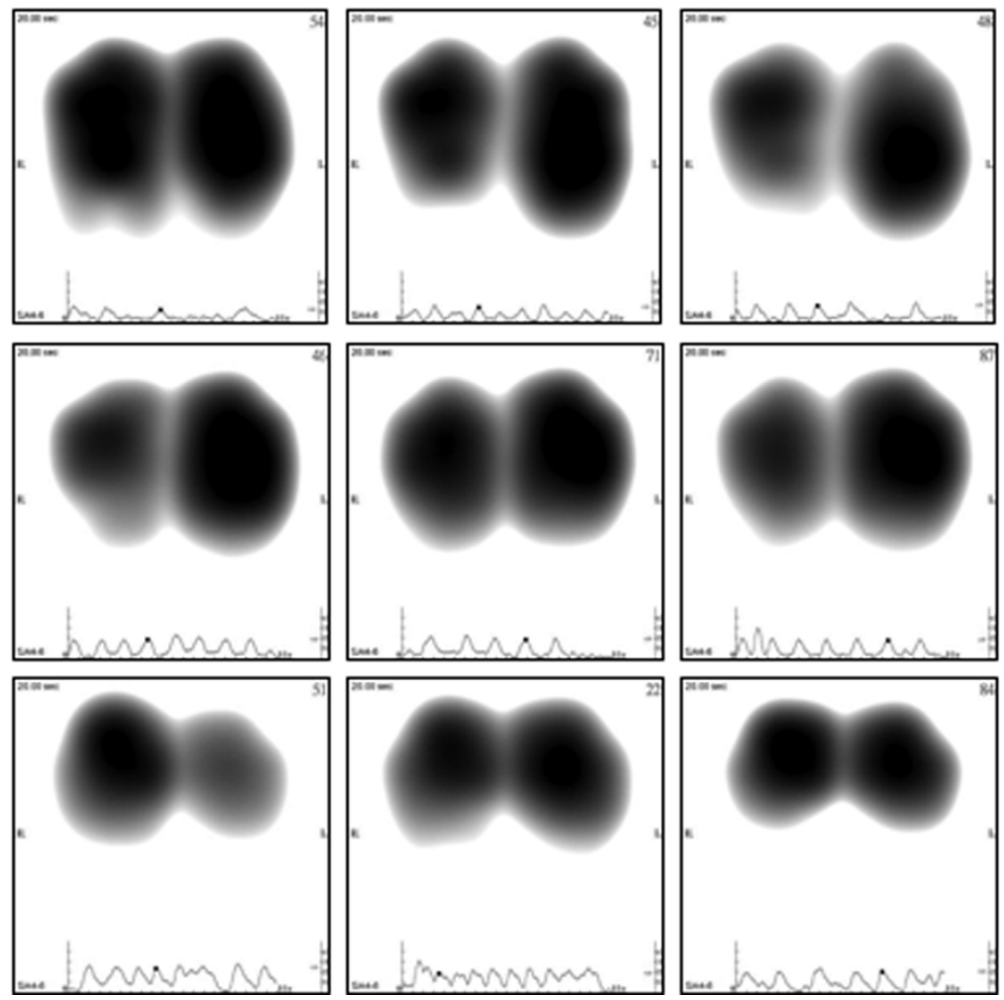
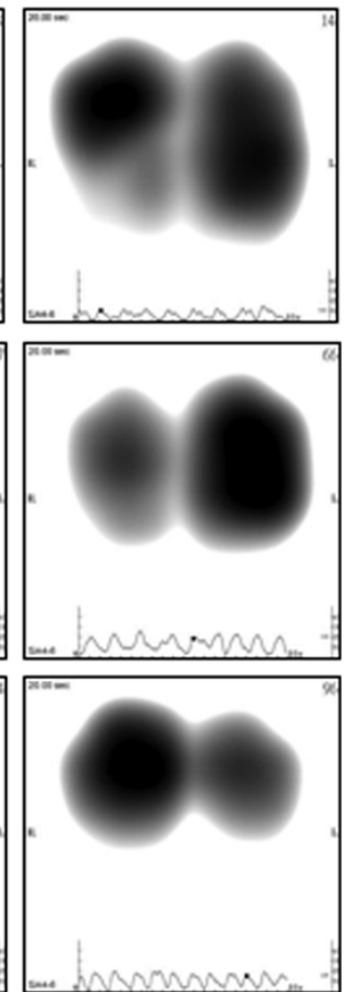

Representative frames (or maps) at peak-inspiratory flow obtained from five individual patients at PEEP levels $0,5,10$ and $15 \mathrm{cmH}_{2} \mathrm{O}$. (a) A 74year-old female with respiratory failure. (b) A 19-year-old male with right pneumothorax. (c) A 83-year-old male with sternal wound infection. (d) A 77-year-old male with myasthenia gravis. (e) A 57-year-old male with acute pancreatitis. PEEP = positive end-expiratory pressure.

may have allowed controlling for VT and inspiratory flow but this was out of the scope of this protocol. Another protocolrelated limitation included the effect of volume history which may interfere with VT distribution. Moreover, differentiation between diaphragmatic redistribution induced by PEEP and VT-induced recruitment may be difficult. The population was heterogeneous and further studies should be performed on a more homogeneous population allocated to specific lung disease categories with emphasis on ALI or ARDS. Moreover, the heterogeneity of the clinical conditions exhibited by the patients at the time of investigation may be a limitation of the present study. Despite normalization, airflow velocity of the ventilators may have affected the results and, considering its impact on VT distribution and dynamic hyperinflation, it would 
Comparison between tidal volume, oxygen saturation, dynamic compliance, and airway resistance at two different levels of apicodiaphragmatic ratio

\begin{tabular}{|c|c|c|c|c|c|c|}
\hline & \multicolumn{3}{|c|}{$\mathrm{RR}<20$ breaths $/$ minute $(15 \pm 3)$} & \multicolumn{3}{|c|}{ RR $>20$ breaths/minute $(26 \pm 5)$} \\
\hline & $\begin{array}{l}\text { ADR }<2 \\
(n=23)\end{array}$ & $\begin{array}{l}A D R>2 \\
(n=14)\end{array}$ & $P$ value & $\begin{array}{l}\text { ADR }<2 \\
(n=17)\end{array}$ & $\begin{array}{l}\text { ADR }>2 \\
(n=23)\end{array}$ & $P$ value \\
\hline $\begin{array}{l}\text { Tidal volume } \\
(\mathrm{ml})\end{array}$ & $577 \pm 91$ & $528 \pm 101$ & NS & $479 \pm 109$ & $483 \pm 135$ & NS \\
\hline $\begin{array}{c}\text { Oxygen saturation } \\
(\%)\end{array}$ & $97 \pm 3$ & $97 \pm 3$ & NS & $97 \pm 3$ & $98 \pm 2$ & NS \\
\hline $\begin{array}{l}\text { Compliance } \\
\text { (mL/mbar) }\end{array}$ & $60 \pm 25$ & $42 \pm 12$ & 0.058 & $51 \pm 25$ & $48 \pm 20$ & NS \\
\hline $\begin{array}{c}\text { Resistance } \\
\text { (mbar L/second) }\end{array}$ & $15 \pm 4$ & $18 \pm 7$ & NS & $15 \pm 3$ & $16 \pm 5$ & NS \\
\hline
\end{tabular}

Apico-diaphragmatic ratio (ADR) was defined as the ratio between the lung sound distribution in the apical lung areas (upper right (UR) + upper left (UL)) and the diaphragmatic lung areas (lower right (LR) + lower left (LL)) (ADR $=(\mathrm{UL}+\mathrm{UR}) /(\mathrm{LL}+\mathrm{LR})$ ). Distribution was considered more heterogeneous if difference was larger than two, threshold derived from experience with healthy patients in supine position. $P$ values are indicated (Wilcoxon two sample test) as well as non-significant (NS) data. RR = respiratory rate.

have been interesting to consider the peak flow values. Furthermore, the protocol did not include a comparison of lung sound distribution with more appropriate tools, such as computerized tomography, functional residual capacity, or electrical impedance tomography. This should be investigated in the future. Although sometimes useful in research and clinical practice $[41,42]$, the reliability of Cdyn is debatable, especially in non-paralyzed patients with non-uniform volume histories.
Despite the fact that during pressure support mode of mechanical ventilation, Cdyn is particularly difficult to interpret, this parameter was used in this study because it was readily accessible in the scope of the protocol. In order to improve the accuracy of the measurement, three values were averaged at each time point. Finally, sound filtering to a band-pass of 150 to $250 \mathrm{~Hz}$ may have reduced the information as lung sound characteristics are contained in other frequency bands, espe-

Figure 6

(a)

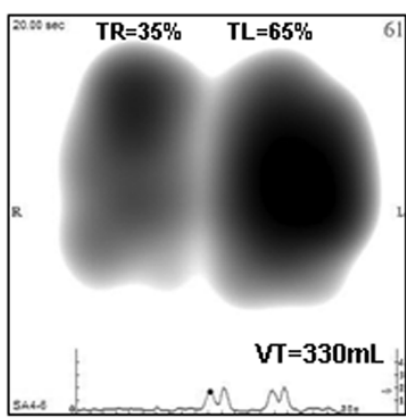

(b)

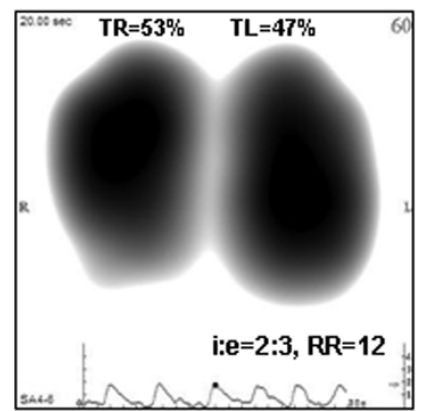

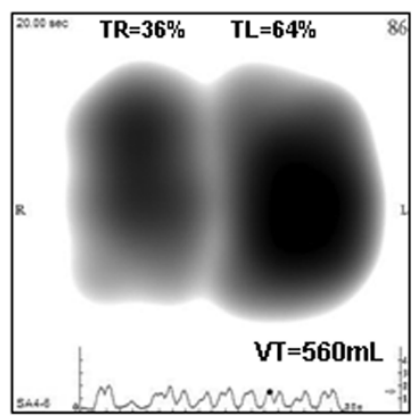

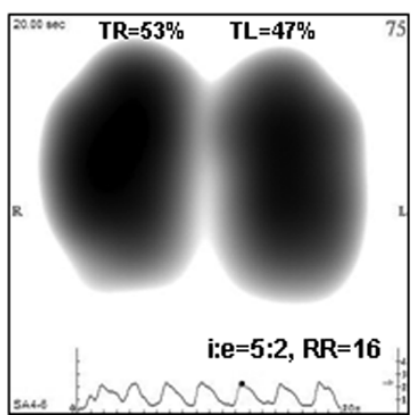

Representative frames (or maps) at peak-inspiratory flow obtained from two patients ventilated with different ventilator settings. (a) A 72-year-old female with chronic obstructive pulmonary disease recorded at positive end-expiratory pressure (PEEP) level of $5 \mathrm{cmH}_{2} \mathrm{O}$ and at two levels of tidal volume (VT; left $=330 \mathrm{~mL}$, right $=560 \mathrm{~mL}$ ); (b) A 24-year-old male with bilateral chest contusion recorded at PEEP level of $7 \mathrm{cmH} \mathrm{C}_{2} \mathrm{O}, \mathrm{VT}$ of $600 \mathrm{~mL}$ and at two levels of respiratory rate (RR) and inspiratory/expiratory ratio (i:e; left: i:e $=2: 3$ and $R R=12$ breaths/minute, right: i:e $=5: 2$ and $R R=16$ breaths/minute). $T L=$ total left lung; $T R=$ total right lung. 
cially above $250 \mathrm{~Hz}$. Notwithstanding these limitations, the development of adjunctive technologies that assist in assessment of clinical benefits of PEEP and recruitment maneuver is still highly desirable $[43,44]$.

\section{Conclusions}

A shift in lung sound distribution from the apical to the diaphragmatic lung areas was observed during PEEP increase. This shift was not correlated with significant change in VT but was associated with an increase in Cdyn. High repeatability was obtained in this population. Further studies are needed in order to elucidate the mechanism of sound shift in relation to PEEP increment and to fully appreciate the contribution of PEEP increase to diaphragmatic sound redistribution.

\section{Key messages}

- Sound distribution shifts from the apical to the diaphragmatic lung areas when increasing level of PEEP from 0 to $10 \mathrm{cmH}_{2} \mathrm{O}$.

- Acoustic shift is not correlated with significant change in VT but is associated with increased Cdyn.

- Sound distribution measurements are highly repeatable in mechanically ventilated patients.

\section{Competing interests}

Research materials for the VRI research program at Rabin Medical Center (Petah Tikva, Israel) are funded partially by Deep Breeze Ltd. SL has consultant agreement that includes honoraria and stock options (no current monetary value) with Deep Breeze Ltd and he was sponsored by GE Healthcare, Deep Breeze's distributor worldwide, to give lectures in academic meetings. YAG is an employee of Deep Breeze Ltd. IK, $\mathrm{DD}, \mathrm{JC}, \mathrm{MG}, \mathrm{MS}$, and PS declare that they have no competing interests.

\section{Authors' contributions}

SL, YAG, IK, DD, MG, and MS participated in the design and coordination of the study and carried out the VRI recordings. SL and YAG worked on the data analysis. SL, YAG, JC, and PS drafted the manuscript. All authors edited and approved the final manuscript.

\section{Acknowledgements}

We would like to express our gratitude to Mrs Michal Kedar for coordinating the data of this study. This research was funded in part by Deep Breeze Ltd.

\section{References}

1. Leblanc P, Macklem PT, Ross WR: Breath sounds and distribution of pulmonary ventilation. Am Rev Respir Dis 1970, 102:10-16.

2. Ploy-Song-Sang Y, Martin RR, Ross WR, Loudon RG, Macklem PT: Breath sounds and regional ventilation. Am Rev Respir Dis 1977, 116:187-199.
3. Ploy-Song-Sang Y, Macklem PT, Ross WR: Distribution of regional ventilation measured by breath sounds. $A m$ Rev Respir Dis 1978, 117:657-664.

4. Kraman SS: The relationship between airflow and lung sound amplitude in normal subjects. Chest 1984, 86:225-229.

5. Gavriely N, Cugell DW: Airflow effects on amplitude and spectral content of normal breath sounds. J Appl Physiol 1996, 80:5-13.

6. Kiyokawa $\mathrm{H}$, Pasterkamp $\mathrm{H}$ : Volume-dependent variations of regional lung sound, amplitude, and phase. J Appl Physiol 2002, 93:1030-1038.

7. Bergstresser T, Ofengeim D, Vyshedskiy A, Shane J, Murphy R: Sound transmission in the lung as a function of lung volume. $J$ Appl Physiol 2002, 93:667-674.

8. Maher TM, Gat M, Allen D, Devaraj A, Wells AU, Geddes DM: Reproducibility of dynamically represented acoustic lung images from healthy individuals. Thorax 2008, 63:542-548.

9. Yigla M, Gat M, Meyer JJ, Friedman PJ, Maher TM, Madison JM: Vibration response imaging technology in healthy subjects. AJR Am J Roentgenol 2008, 191:845-852.

10. Murphy RL, Vyshedskiy A, Power-Charnitsky VA, Bana DS, Marinelli PM, Wong-Tse A, Paciej R: Automated lung sound analysis in patients with pneumonia. Respir Care 2004, 49:1490-1497.

11. Mor R, Kushnir I, Meyer JJ, Ekstein J, Ben-Dov I: Breath sound distribution images of patients with pneumonia and pleural effusion. Respir Care 2007, 52:1753-1760.

12. Kramer MR, Raviv $Y$, Hardoff $R$, Shteinmatz A, Amital A, Shitrit $D$ : Regional breath sound distribution analysis in single-lung transplant recipients. J Heart Lung Transplant 2007, 26:1149-1154.

13. Räsenen J, Gavriely N: Detection of porcine oleic acid-induced acute lung injury using pulmonary acoustics. I Appl Physiol 2002, 93:51-57.

14. Räsenen J, Gavriely N: Response of acoustic transmission to positive airway pressure therapy in experimental lung injury. Intensive Care Med 2005, 31:1434-1441.

15. Vena A, Perchiazzi, Giuliani R, Fiore T, Hedenstierna G: Acoustic effects of positive end-expiratory pressure on normal lung sounds in mechanically ventilated pigs. Clin Physiol Funct Imaging 2006, 26:45-53.

16. Peták F, Habre W, Babik B, Tolnai J, Hantos Z: Crackle-sound recording to monitor airway closure and recruitment in ventilated pigs. Eur Respir J 2006, 27:808-816.

17. Vena A, Perchiazzi G, Rylander C, Giuliani R, Fiore T, Magnusson A, Hedenstierna : Breath sound analysis detects injury and recruitment in the lung during mechanical ventilation. Intensive Care Med 2008, 34(Suppl 1):549.

18. Hubmayr RD: The times are a-changin' should we hang up the stethoscope? Anesthesiology 2004, 100:1-2.

19. Lichtenstein D, Goldstein I, Mourgeon E, Cluzel P, Grenier P, Rouby J-J: Comparative diagnostic performances of auscultation, chest radiography, and lung ultrasonography in acute respiratory distress syndrome. Anesthesiology 2004, 100:9-15

20. Cinel I, Jean S, Dellinger RP: Dynamic lung imaging techniques in mechanically ventilated patients. In Yearbook of Intensive Care and Emergency Medicine Edited by: Vincent JL. Heidelberg: Springer-Verlag; 2007:373-380

21. Lev $S$, Singer $P$, Glickman $Y A$ : Vibration response imaging: a novel technology for lung monitoring in critically ill patients. In Yearbook of Respiratory Care Clinics and Applied Technologies Edited by: Esquinas A. Murcia: World Federation of Respiratory Care and Applied Technologies; 2008:530-539.

22. Waitman LR, Clarkson KP, Barwise JA, King PH: Representation and classification of breath sounds recorded in an intensive care setting using neural networks. J Clin Monit Comput 2000, 16:95-105.

23. Prodhan $\mathrm{P}$, Dela Rosa RS, Shubina M, Haver KE, Matthews BD, Buck S, Kacmarek RM, Noviski NN: Wheeze detection in the pediatric intensive care unit: comparison among physician, nurses, respiratory therapists, and a computerized respiratory sound monitor. Respir Care 2008, 53:1304-1309.

24. Tejman-Yarden S, Lederman D, Eilig I, Zlotnik A, Weksler N, Cohen A, Gurman GM: Acoustic monitoring of double-lumen ventilated lungs for the detection of selective unilateral lung ventilation. Anesth Analg 2006, 103:1489-1493. 
25. Tejman-Yarden S, Zlotnik A, Weizman L, Tabrikian J, Cohen A, Weksler N, Gurman GM: Acoustic monitoring of lung sounds for the detection of one-lung intubation. Anesth Analg 2007, 105:397-404.

26. Jean S, Cinel I, Gratz I, Tay C, Lotano V, Deal E, Parillo JE, Dellinger RP: Image-based monitoring of one-lung ventilation. Eur $J$ Anaesthesio/ 2008, 20:1-7.

27. Cinel I, Jean S, Tay C, Gratz I, Deal E, Parillo JE, Dellinger RP: Case report: vibration response imaging findings following inadvertent esophageal intubation. Can J Anaesth 2008, 55:172-176.

28. Dellinger RP, Jean S, Cinel I, Tay C, Rajanala S, Glickman YA, Parillo JE: Regional distribution of acoustic-based lung vibration as a function of mechanical ventilation mode. Critical Care 2007, 11:R26.

29. Trivedi H, Cinel I, Jean S, Tay C, Durflinger P, Parillo JE, Dellinger $\mathrm{RP}$ : The role of vibration response imaging in the titration of PEEP in a mechanically ventilated patient with acute respiratory distress syndrome. Crit Care Med 2007, 34(Suppl 12):608.

30. Cinel I, Dellinger RP, Jean S, Glickman YA, Parillo JE: Assessment of the effectiveness of lung recruitment and PEEP setting by vibration response imaging. Crit Care 2006, 10(Suppl 1):15.

31. Lev S, Kagan I, Grinev M, Cohen J, Singer P: Positive end-expiratory pressure-induced changes of the vibration response image. Crit Care 2008, 12(Suppl 2):299.

32. Lev S, Cohen J, Kagan I, Grinev M, Singer P: Lung sound distribution shifts to the lower lung regions with increased PEEP. Intensive Care Med 2008, 34(Suppl 1):545.

33. Talmor D, Sarge T, Malhotra A, O'Donnell CR, Ritz R, Lisbon A, Novack V, Loring SH: Mechanical ventilation guided by esophageal pressure in acute lung injury. N Engl J Med 2008, 359:2095-104.

34. Pelosi P, Caironi P, Bottino N, Gattinoni L: Positive end expiratory pressure in anesthesia. Minerva Anestesiol 2000, 66:297-306.

35. Nieszkowska A, Lu Q, Vieira S, Elman M, Fetita C, Rouby JJ: Incidence and regional distribution of lung overinflation during mechanical ventilation with positive end-expiratory pressure. Crit Care Med 2004, 32:1496-1503.

36. Dellinger PR, Parrillo JE, Kushnir A, Rossi M, Kushnir I: Dynamic visualization of lung sounds with a vibration response device: a case series. Respiration 2008, 75:60-72.

37. Bentur L, Livnat G, Husein D, Pollack S, Rotschild M: Dynamic visualization of breath sounds distribution in suspected foreign body aspiration. J Bronchology 2007, 14:156-161.

38. Jean S, Cinel I, Tay C, Parrillo JE, Dellinger RP: Assessment of asymmetric lung disease in intensive care unit patients using vibration response imaging. Anesth Analg 2008, 107:1243-1247.

39. Mahagnah M, Gavriely N: Repeatability of measurements of normal lung sounds. Am J Respir Crit Care Med 1994, 149:477-481.

40. Sánchez I, Vizcaya C: Tracheal and lung sounds repeatability in normal adults. Respir Med 2003, 97:1257-1260.

41. Suarez-Sipmann F, Böhm SH, Tusman G, Pesch T, Thamm O, Reissmann H, Reske A, Magnusson A, Hedenstierna G: Use of dynamic compliance for open lung positive end-expiratory pressure titration in an experimental study. Crit Care Med 2007, 35:214-221.

42. Suarez-Sipmann F: Titrating open lung PEEP in acute lung injury. A clinical method based on changes in dynamic compliance. In Digital Comprehensive Summaries of Uppsala Dissertations from the Faculty of Medicine 313 Acta Universitatis Upsaliensis, Uppsala; 2008.

43. Mercat A, Richard JC, Vielle B, Jaber S, Osman D, Diehl JL, Lefrant JY, Prat G, Richecoeur J, Nieszkowska A, Gervais C, Baudot J, Bouadma L, Brochard L, Expiratory Pressure (Express) Study Group: Positive end-expiratory pressure setting in adults with acute lung injury and acute respiratory distress syndrome: a randomized controlled trial. JAMA 2008, 299:646-655.

44. Meade MO, Cook DJ, Guyatt GH, Slutsky AS, Arabi YM, Cooper DJ, Davies AR, Hand LE, Zhou Q, Thabane L, Austin P, Lapinsky S, Baxter A, Russell J, Skrobik Y, Ronco JJ, Stewart TE, Lung Open Ventilation Study Investigators: Ventilation strategy using low tidal volumes, recruitment maneuvers, and high positive endexpiratory pressure for acute lung injury and acute respiratory distress syndrome: a randomized controlled trial. JAMA 2008, 299:637-645. 